\title{
From discrete particles to continuum fields near a boundary
}

\author{
Thomas Weinhart • Anthony R. Thornton • \\ Stefan Luding • Onno Bokhove
}

Received: 22 August 2011 / Published online: 14 February 2012

(C) The Author(s) 2012. This article is published with open access at Springerlink.com

\begin{abstract}
An expression for the stress tensor near an external boundary of a discrete mechanical system is derived explicitly in terms of the constituents' degrees of freedom and interaction forces. Starting point is the exact and general coarse graining formulation presented by Goldhirsch (Granul Mat 12(3):239-252, 2010), which is consistent with the continuum equations everywhere but does not account for boundaries. Our extension accounts for the boundary interaction forces in a self-consistent way and thus allows the construction of continuous stress fields that obey the macroscopic conservation laws even within one coarse-graining width of the boundary. The resolution and shape of the coarse-graining function used in the formulation can be chosen freely, such that both microscopic and macroscopic effects can be studied. The method does not require temporal averaging and thus can be used to investigate time-dependent flows as well as static or steady situations. Finally, the fore-mentioned continuous field can be used to define 'fuzzy' (very rough) boundaries. Discrete particle simulations are presented in which the novel boundary treatment is exemplified, including chute flow over a base with roughness greater than one particle diameter.
\end{abstract}

T. Weinhart $(\bowtie) \cdot$ A. R. Thornton $\cdot$ S. Luding

Multiscale Mechanics, Department of Mechanical Engineering, University of Twente, P.O. Box 217, 7500 AE Enschede,

The Netherlands

e-mail: t.weinhart@utwente.nl

T. Weinhart · A. R. Thornton · O. Bokhove

Numerical Analysis and Computation Mechanics,

Department of Applied Mathematics, University of Twente,

P.O. Box 217, 7500 AE Enschede, The Netherlands
Keywords Coarse graining - Averaging - Boundary treatment · DPM (DEM) · Discrete mechanical systems . Homogenisation · Stress - Continuum mechanics . Granular systems

\section{Introduction}

The main topic of this paper is the issue of coarse-graining, near a boundary. We consider the bulk method described by Goldhirsch [1], and extend it to account for boundary forces due to the presence of a wall or base. The Goldhirsch special edition of Granular Matter may be the appropriate place to present some of the ideas that we have developed in this area based on those of Isaac Goldhirsch.

Continuum fields often need to be constructed from discrete particle data. In molecular dynamics [2] and granular systems [3,4], these discrete data are the positions, velocities of, and forces on, each atom or particle. In contrast, in the case of smooth particle hydrodynamics [5], the continuum system itself is approximated by a discrete set of fluid parcels. In all these methods, a crucially important issue is how to compute the continuum fields in the most appropriate way. Several techniques have been developed to calculate the continuum fields, see [6] and references therein. Particularly the stress tensor is of interest: the techniques include the IrvinKirkwood's approach [7] or the method of planes [8]. Here, we use the coarse-graining approach (CG) as described in Refs. [1,9].

The CG method [1,9] has several advantages over other methods, including: (i) the fields automatically satisfy the conservation equations of continuum mechanics; (ii) it is not assumed that the particles are rigid or spherical, and (iii) the results are valid for a single configuration of particles (no averaging over ensembles of particles is required). The only 
assumptions are: each particle pair has a single point of contact, the contact area can be replaced by a contact point, and collisions are not instantaneous.

In Sect. 2, we use the derivation of Goldhirsch [1] to extend the CG method to account for the presence of a boundary. Explicit expressions for the resulting continuum fields are derived. In Sect. 2.5, an alternative stress definition is proposed extending the stress field into the boundary region. In Sect. 3, the approach is tested with two DPM simulations, and in Sect. 4 we draw conclusions.

\section{Theory}

\subsection{Assumptions and notation}

We are interested in deriving macroscopic fields, such as density, velocity and the stress tensor from averages of microscopic variables such as the positions, velocities of, and forces on, the constituents. Averaging will be done such that the continuum fields, by construction, satisfy conservation laws. Vectorial and tensorial components are denoted by Greek letters in order to distinguish them from the Latin particle-indices $i, j$. Bold vector notation will be used when appropriate. We will follow the derivation of Goldhirsch [1], but extend it by introducing two types of particle: $N$ flowing particles $\{1,2, \ldots, N\}$ and $K$ boundary particles $\{N+1, \ldots, N+K\}$.

Each particle $i$ has mass $m_{i}$, centre of mass position $r_{i \alpha}$ and velocity $v_{i \alpha}$. The force $f_{i \alpha}$ acting on particle $i$ is a combination of the sum of the interaction force $f_{i j \alpha}$ with another particle $j$, the interaction force $f_{i k \alpha}$ with a boundary particle $k$, and a body force $b_{i \alpha}$ (e.g., gravity),

$f_{i \alpha}=\sum_{j=1, j \neq i}^{N} f_{i j \alpha}+\sum_{k=N+1}^{N+K} f_{i k \alpha}+b_{i \alpha}, \quad i \leq N$.

The interaction forces are binary and anti-symmetric such that action equals reaction, $f_{i j \alpha}=-f_{j i \alpha}, i, j \leq N$. We assume that each particle pair $(i, j), i \leq N, j \leq N+K$ has, at most, a single contact point, $c_{i j \alpha}$, at which the contact forces act. The positions of the boundary particles are fixed, as if they had infinite mass. The trajectories of the flowing particles are governed by Newton's second law and if tangential forces and torques are present, rotations follow from the angular form of Newton's law.

In the following sections, we commence from Ref. [1] to derive definitions of the continuum fields. To be precise, a body force density is introduced to account for body forces, and to incorporate boundary effects an interaction force density (IFD) is introduced. While the idea of an IFD is more generally applicable (e.g., for mixtures), it is employed here to account for the presence of a boundary.

\subsection{Coarse graining}

From statistical mechanics, the microscopic mass density of the flow at a point $r_{\alpha}$ at time $t$ is defined by

$\rho^{\mathrm{mic}}(\boldsymbol{r}, t)=\sum_{i=1}^{N} m_{i} \delta\left(\boldsymbol{r}-\boldsymbol{r}_{i}(t)\right)$

where $\delta(\boldsymbol{r})$ is the Dirac delta function. We use the following definition of the macroscopic density,

$\rho(\boldsymbol{r}, t)=\sum_{i=1}^{N} m_{i} \mathscr{W}\left(\boldsymbol{r}-\boldsymbol{r}_{i}(t)\right)$,

i.e., we have replaced the Dirac delta function by an integrable 'coarse-graining' function $\mathscr{W}$ whose integral over the domain is unity and has a predetermined width, or coarsegraining scale, $w$.

\subsection{Mass balance}

The coarse-grained momentum density is defined by

$p_{\alpha}(\boldsymbol{r}, t)=\sum_{i=1}^{N} m_{i} v_{i \alpha} \mathscr{W}\left(\boldsymbol{r}-\boldsymbol{r}_{i}\right)$

The macroscopic velocity field $V_{\alpha}(\boldsymbol{r}, t)$ is defined as the ratio of momentum and density fields, $V_{\alpha}(\boldsymbol{r}, t)=p_{\alpha}(\boldsymbol{r}, t) / \rho(\boldsymbol{r}, t)$. It is straightforward to confirm that $\rho_{\alpha}$ and $p_{\alpha}$ satisfy the continuity equation (c.f. [1,9]),

$\frac{\partial \rho}{\partial t}+\frac{\partial p_{\alpha}}{\partial r_{\alpha}}=0$

\subsection{Momentum balance}

Subsequently, we will consider the momentum conservation equation with the aim of establishing the macroscopic stress field, $\sigma_{\alpha \beta}$. As we want to describe boundary stresses as well as internal stresses, the boundary interaction force density (IFD), $t_{\alpha}$, has been included, as well as the body force density, $b_{\alpha}$, which are not present in the original derivation [1]. The desired momentum balance equations take the form,

$\frac{\partial p_{\alpha}}{\partial t}=-\frac{\partial}{\partial r_{\beta}}\left[\rho V_{\alpha} V_{\beta}\right]+\frac{\partial \sigma_{\alpha \beta}}{\partial r_{\beta}}+t_{\alpha}+b_{\alpha}$.

To determine the stress it is required to compute the temporal derivative of (4),

$\frac{\partial p_{\alpha}}{\partial t}=\sum_{i=1}^{N} f_{i \alpha} \mathscr{W}\left(\boldsymbol{r}-\boldsymbol{r}_{i}\right)+\sum_{i=1}^{N} m_{i} v_{i \alpha} \frac{\partial}{\partial t} \mathscr{W}\left(\boldsymbol{r}-\boldsymbol{r}_{i}\right)$ 
where $f_{i \alpha}=m_{i} \mathrm{~d} v_{i \alpha} / \mathrm{d} t$ is the total force on particle $i$. Using (1), the first term in (7) can be expanded as

$A_{\alpha} \equiv \sum_{i=1}^{N} \sum_{j=1, j \neq i}^{N} f_{i j \alpha} \mathscr{W}_{i}+\sum_{i=1}^{N} \sum_{k=N+1}^{N+K} f_{i k \alpha} \mathscr{W}_{i}+\sum_{i=1}^{N} b_{i \alpha} \mathscr{W}_{i}$

with the abbreviation $\mathscr{W}_{i}=\mathscr{W}\left(\boldsymbol{r}-\boldsymbol{r}_{i}\right)$. The first term, which represents the bulk particle interactions, satisfies

$$
\begin{aligned}
\sum_{i=1}^{N} \sum_{j=1, j \neq i}^{N} f_{i j \alpha} \mathscr{W}_{i} & =\sum_{i=1}^{N} \sum_{j=1, j \neq i}^{N} f_{j i \alpha} \mathscr{W}_{j} \\
& =-\sum_{i=1}^{N} \sum_{j=1, j \neq i}^{N} f_{i j \alpha} \mathscr{W}_{j}
\end{aligned}
$$

since $f_{i j \alpha}=-f_{j i \alpha}$ and because the dummy summation indices can be interchanged. It follows from (9) that

$$
\begin{aligned}
\sum_{i=1}^{N} \sum_{j=1, j \neq i}^{N} f_{i j \alpha} \mathscr{W}_{i} & =\frac{1}{2} \sum_{i=1}^{N} \sum_{j=1, j \neq i}^{N} f_{i j \alpha}\left(\mathscr{W}_{i}-\mathscr{W}_{j}\right) \\
& =\sum_{i=1}^{N} \sum_{j=i+1}^{N} f_{i j \alpha}\left(\mathscr{W}_{i}-\mathscr{W}_{j}\right)
\end{aligned}
$$

Substituting (10) into (8) yields

$$
A_{\alpha}=\sum_{i=1}^{N} \sum_{j=i+1}^{N} f_{i j \alpha}\left(\mathscr{W}_{i}-\mathscr{W}_{j}\right)+\sum_{i=1}^{N} \sum_{k=N+1}^{N+K} f_{i k \alpha} \mathscr{W}_{i}+b_{\alpha}
$$

where $b_{\alpha}=\sum_{i} b_{i \alpha} \mathscr{W}_{i}$ is the body force density.

Next, $A_{\alpha}$ is rewritten using the chain rule and Leibnitz's integral rule to obtain a formula for the stress tensor. The following identity holds for any continuously differentiable coarse-graining function $\mathscr{W}$

$$
\begin{aligned}
\mathscr{W}_{j}-\mathscr{W}_{i} & =\int_{0}^{1} \frac{\partial}{\partial s} \mathscr{W}\left(\boldsymbol{r}-\boldsymbol{r}_{i}+s \boldsymbol{r}_{i j}\right) \mathrm{d} s \\
& =r_{i j \beta} \frac{\partial}{\partial r_{\beta}} \int_{0}^{1} \mathscr{W}\left(\boldsymbol{r}-\boldsymbol{r}_{i}+s \boldsymbol{r}_{i j}\right) \mathrm{d} s,
\end{aligned}
$$

where $r_{i j \alpha}=r_{i \alpha}-r_{j \alpha}$ is the vector from $r_{j \alpha}$ to $r_{i \alpha}$.

Substituting identities (12) into (11) yields

$$
\begin{aligned}
A_{\alpha}= & -\frac{\partial}{\partial r_{\beta}} \sum_{i=1}^{N} \sum_{j=i+1}^{N} f_{i j \alpha} r_{i j \beta} \int_{0}^{1} \mathscr{W}\left(\boldsymbol{r}-\boldsymbol{r}_{i}+s \boldsymbol{r}_{i j}\right) \mathrm{d} s \\
& +\sum_{i=1}^{N} \sum_{k=N+1}^{N+K} f_{i k \alpha} \mathscr{W}_{i}+b_{\alpha} .
\end{aligned}
$$

In Ref. [1], it is shown that the second term in (7) can be expressed as

$$
\sum_{i=1}^{N} m_{i} v_{i \alpha} \frac{\partial}{\partial t} \mathscr{W}_{i}=-\frac{\partial}{\partial r_{\beta}}\left[\rho V_{\alpha} V_{\beta}+\sum_{i=1}^{N} m_{i} v_{i \alpha}^{\prime} v_{i \beta}^{\prime} \mathscr{W}_{i}\right]
$$

where $v_{i \alpha}^{\prime}$ is the fluctuation velocity of particle $i$, given by

$v_{i \alpha}^{\prime}(\boldsymbol{r}, t)=v_{i \alpha}(t)-V_{\alpha}(\boldsymbol{r}, t)$.

Substituting (13) and (14) into momentum balance (6) yields

$$
\frac{\partial \sigma_{\alpha \beta}}{\partial r_{\beta}}+t_{\underline{\alpha}}=\frac{\partial \sigma_{\alpha \beta}^{k}}{\partial r_{\beta}}+\frac{\partial \sigma_{\alpha \beta}^{b}}{\partial r_{\beta}}+\sum_{i=1}^{N} \sum_{k=N+1}^{N+K} f_{i k \alpha} \mathscr{W}_{i}
$$

where the kinetic and bulk contact contributions to the stress tensor are defined as

$$
\begin{aligned}
\sigma_{\alpha \beta}^{k} & =-\sum_{i=1}^{N} m_{i} v_{i \alpha}^{\prime} v_{i \beta}^{\prime} \mathscr{W}_{i} \\
\sigma_{\alpha \beta}^{b} & =-\sum_{i=1}^{N} \sum_{j=i+1}^{N} f_{i j \alpha} r_{i j \beta} \int_{0}^{1} \mathscr{W}\left(\boldsymbol{r}-\boldsymbol{r}_{i}+s \boldsymbol{r}_{i j}\right) \mathrm{d} s .
\end{aligned}
$$

Here, the underlined terms in (16) are not in the original derivation presented in Ref. [1] and account for the presence of the boundary.

Expression (16) can be satisfied by defining the last term on the right hand side as the interaction force density (IFD). This however has the disadvantage that the boundary IFD is located around the centre of mass of the flowing particles. The more natural physical location of the boundary IFD would be at the interface between the flowing and boundary particles.

Therefore, we move the IFD to the contact points, $c_{i k \alpha}$, between flowing and boundary particles: similar to (12),

$\mathscr{W}_{i k}-\mathscr{W}_{i}=a_{i k \beta} \frac{\partial}{\partial r_{\beta}} \int_{0}^{1} \mathscr{W}\left(\boldsymbol{r}-\boldsymbol{r}_{i}+s \boldsymbol{a}_{i k}\right) \mathrm{d} s$,

where $\mathscr{W}_{i k}=\mathscr{W}\left(\boldsymbol{r}-\boldsymbol{c}_{i k}\right)$ and $a_{i k \alpha}=r_{i \alpha}-c_{i k \alpha}$. Substituting (18) into the last term in (16) we obtain

$$
\begin{aligned}
& \sum_{i=1}^{N} \sum_{k=N+1}^{N+K} f_{i k \alpha} \mathscr{W}_{i}=\sum_{i=1}^{N} \sum_{k=N+1}^{N+K} f_{i k \alpha} \mathscr{W}_{i k} \\
& \quad-\frac{\partial}{\partial r_{\beta}}\left[\sum_{i=1}^{N} \sum_{k=N+1}^{N+K} f_{i k \alpha} a_{i k \beta} \int_{0}^{1} \mathscr{W}\left(\boldsymbol{r}-\boldsymbol{r}_{i}+s \boldsymbol{a}_{i k}\right) \mathrm{d} s\right] .
\end{aligned}
$$

Thus, substituting (19) into (16), we define the stress by

$$
\sigma_{\alpha \beta}=\sigma_{\alpha \beta}^{k}+\sigma_{\alpha \beta}^{b}+\sigma_{\alpha \beta}^{w},
$$


where the contribution to the stress from the contacts between flow and boundary particles is

$\sigma_{\alpha \beta}^{w}=-\sum_{i=1}^{N} \sum_{k=N+1}^{N+K} f_{i k \alpha} a_{i k \beta} \int_{0}^{1} \mathscr{W}\left(\boldsymbol{r}-\boldsymbol{r}_{i}+s \boldsymbol{a}_{i k}\right) \mathrm{d} s$,

and the IFD on the left hand side of Eq. (16) is

$t_{\alpha}=\sum_{i=1}^{N} \sum_{k=N+1}^{N+K} f_{i k \alpha} \mathscr{W}_{i k}$.

Equations (20) differ from the standard result of [1] by an extra term, $\sigma_{\alpha \beta}^{w}$, that accounts for the additional stress created by the interaction of the boundary with the flow. The definition (21) gives the boundary IFD applied by the flowing particles; i.e., it has been constructed such that in the limit $w \rightarrow 0$, the IFD acts at the contact points between boundary and flow. Note that for a finite coarse-graining width, the domain of the coarse-grained fields would extend beyond the domain enclosed by (rigid) walls or boundaries.

Note that this framework is general and can be used to compute more than boundary IFDs. For example, one can obtain the drag between two different species of interacting particles by replacing the flowing and boundary particles with the particles of the two species in the definition of the continuum fields. By placing an IFD at the contact points, the IFDs of both species are exactly antisymmetric and thus disappear in the momentum continuity equation of the combined system. In mixture theory, e.g., [10], such interaction terms appear in the governing equations for the individual constituents and are called interaction body forces. These interaction body forces are an exact analog to the IFDs. Therefore, our approach can interpreted as treating the system as a mixture of boundary and flow particles and the IFD is the interaction body force between different species of particle.

Further, we note that the integral of the stress in (17) and (20) over the domain $\Omega$ satisfies the virial definition of mechanical stress,

$$
\begin{aligned}
\int_{\Omega} \sigma_{\alpha \beta} \mathrm{d} \boldsymbol{r} & =-\sum_{i=1}^{N} m_{i} v_{i \alpha}^{\prime} v_{i \beta}^{\prime} \\
- & \sum_{i=1}^{N} \sum_{j=i+1}^{N} f_{i j \alpha} r_{i j \beta}-\sum_{i=1}^{N} \sum_{k=N+1}^{N+K} f_{i k \alpha} a_{i k \beta} .
\end{aligned}
$$

\subsection{Extending the stress profile into a base or wall}

In contrast to the previous subsection, an alternative stress definition is presented here, where the IFD and the stress are combined into a single tensor. Similar to (12) and (18), the following identity holds,

$$
\begin{aligned}
-\mathscr{W}_{i} & =\int_{0}^{\infty} \frac{\partial}{\partial s} \mathscr{W}\left(\boldsymbol{r}-\boldsymbol{r}_{i}+s \boldsymbol{r}_{i k}\right) \mathrm{d} s \\
& =r_{i k \beta} \frac{\partial}{\partial r_{\beta}} \int_{0}^{\infty} \mathscr{W}\left(\boldsymbol{r}-\boldsymbol{r}_{i}+s \boldsymbol{r}_{i k}\right) \mathrm{d} s,
\end{aligned}
$$

since the coarse-graining function $\mathscr{W}$ satisfies $\mathscr{W}(|\boldsymbol{r}| \rightarrow$ $\infty)=0$. Substituting (23) into (16) we can obtain an alternative solution with zero IFD, $t_{\alpha}^{\prime}=0$, where the stress is given by $\sigma_{\alpha \beta^{\prime}}=\sigma_{\alpha \beta}^{k}+\sigma_{\alpha \beta}^{b}+\sigma_{\alpha \beta}^{w}{ }^{\prime}$, with

$\sigma_{\alpha \beta}^{w \prime}=-\sum_{i=1}^{N} \sum_{k=N+1}^{N+K} f_{i k \alpha} r_{i k \beta} \int_{0}^{\infty} \mathscr{W}\left(\boldsymbol{r}-\boldsymbol{r}_{i}+s \boldsymbol{r}_{i k}\right) \mathrm{d} s$.

This stress definition is not identical to the one in (20) and (17). It eliminates the IFD term entirely and provides a natural extension of the stress into the boundary. However, the extended stress does contain contributions from both internal and external forces, and the spatial integral of the stress components has to be extended to infinity. In singular special cases this can lead to artificial results. Another disadvantage of Eq. (24) is its difficult interpretation due to the long-ranging integral. One could see it as the stress inside a 'virtual' wall-material on which the body-force is not acting (equivalent to virtual foam with zero mass-density). However, this is not physical, hence, we prefer the formulation in Sect. 2.4.

It is also possible to extend the stress tensor to the boundary by other means, such as mirroring the stress at the boundary, or using a one-sided coarse-graining function. This is not discussed further since the first method requires a definition of the exact location of the boundary, while the second method can introduce a spatial shift in the stress field due to spatial inhomogeneities.

\section{Results}

\subsection{Contact model}

For illustrational purposes, we simulate a granular system with contact interaction forces. The coarse-graining method, however, is based only on the assumptions in Sect. 2 and therefore can be applied more generally. We use a viscoelastic force model with sliding friction as described in detail in $[4,11]$. The parameters of the system are nondimensionalised such that the flow particle diameters are $d=1$, their mass $m=1$, and the magnitude of gravity $g=1$. The normal spring and damping constants are $k^{n}=2 \cdot 10^{5}$ and $\gamma^{n}=$ 50 , respectively; thus, the collision time is $t_{c}=0.005 \sqrt{d / g}$ and the coefficient of restitution is $\epsilon=0.88$. The tangential spring and damping constants are $k^{t}=(2 / 7) k_{n}$ and $\gamma^{t}=\gamma^{n}$, such that the frequency of normal and tangential 


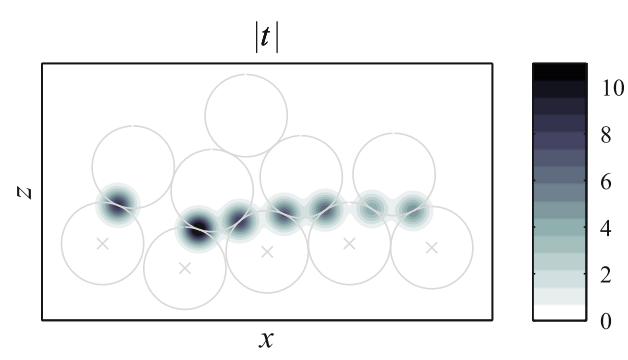

Fig. 1 Gray circles denote a two-dimensional configuration of free and fixed boundary particles, with gravity in the $z$-direction. Fixed particles are marked with a cross in the center. Contour plots show the spatial distribution of the norms of the boundary IFD, $|\boldsymbol{t}|=\sqrt{t_{\alpha}^{2}}$, and the con-

contact oscillations, and the normal and tangential dissipation are equal. The microscopic friction coefficient is set to $\mu^{p}=0.5$. We integrate the resulting force and torque relations in time using the Velocity-Verlet and Euler algorithm, respectively, with a time step $\Delta t=t_{c} / 50$.

We take the coarse-graining function to be a Gaussian of width, or variance, $w$. Other coarse-graining functions are allowed, but the Gaussian has the advantage that it produces smooth fields and the required integrals can be treated analytically.

\subsection{Quasi-static example in two dimensions}

In order to visualise definitions (20) and (21), we firstly consider a two-dimensional configuration consisting of five fixed boundary particles and five flowing bulk particles, with gravity in the $z$-direction, see Fig. 1. The flow is relaxed until the flowing particles are static; hence, the only contribution to the stress is due to the enduring contacts. To visualise the spatial distribution of the IFD and stress, the norms $|\boldsymbol{t}|=\sqrt{t_{\alpha}^{2}}$ and $|\boldsymbol{\sigma}|=\max _{|\boldsymbol{x}|=1}|\boldsymbol{\sigma} \boldsymbol{x}|$ (the maximum absolute eigenvalue), are displayed in Fig. 1. A very small coarse graining width, $w=d / 8$, is chosen to make the spatial averaging visible: the IFD, Eq. (21), centres around the contact points between flowing and static particles, $r_{i k \alpha}$, while the stress, Eqs. (17) and (20), is distributed along the contact lines, $\overline{r_{i \alpha} r_{j \alpha}}$ and $\overline{r_{i \alpha} c_{i k \alpha}}$.

\subsection{Three-dimensional steady chute flow}

Secondly, we consider a three-dimensional simulation of a steady uniform granular chute flow, see Fig. 2 and Ref. [12]. The chute is periodic in the $x$ - and $y$-directions and has dimensions $(x, y) \in[0,20] \times[0,10]$. The chute is inclined at $\theta=26^{\circ}$ and the bed consists of a disordered, irregular boundary created from fixed particles with size $d_{\text {base }}=2$. The chute contains 1000 flowing particles, which are initially randomly distributed. The simulation is computed until

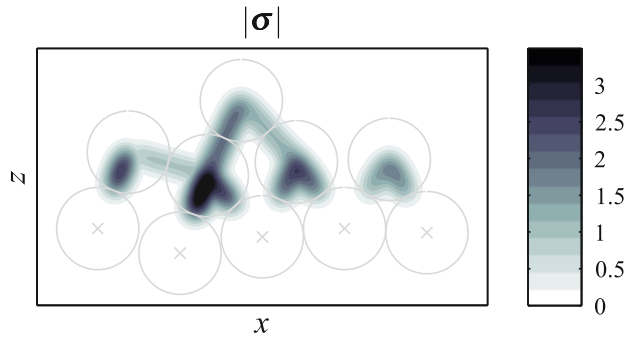

tact stress, $|\boldsymbol{\sigma}|=\max _{|\boldsymbol{x}|=1}|\boldsymbol{\sigma} \boldsymbol{x}|$. A very small coarse graining width, $w=d / 8$, is chosen to make the spatial averaging visible: the IFD centers around the contact point, while the stress is distributed along the contact lines

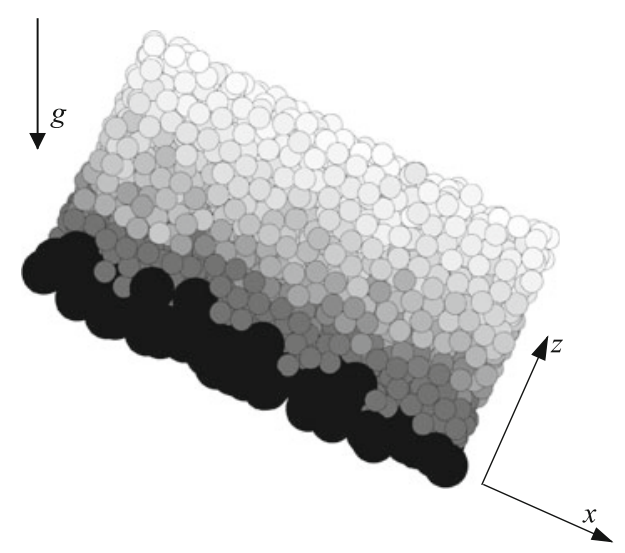

Fig. 2 Steady chute flow over a very rough frictional surface of inclination $\theta=26^{\circ}$ for $N=1,000$ flowing particles. Gravity direction $\boldsymbol{g}$ and coordinates $(x, y, z)$ as indicated. The domain is periodic in the $x$-and $y$-directions. The shading indicates the speed; dark is slow and bright is fast

a steady state is reached. A screen shot of the steady-state system is given in Fig. 2.

Depth profiles for steady uniform flow are obtained by averaging with a coarse-graining width $w=d / 4$ over $x \in$ $[0,20], y \in[0,10]$ and $t \in[2000,2100]$. The spatial averaging is done analytically, while we average in time with snapshots taken every $t_{c} / 2$.

Note that the stress definitions (20) and (24) satisfy

$\frac{\partial \sigma_{\alpha \beta}^{\prime}}{\partial r_{\beta}}=\frac{\partial \sigma_{\alpha \beta}}{\partial r_{\beta}}+t_{\alpha}$

After averaging over time and in the $x$ and $y$ directions, this yields

$\frac{\partial \sigma_{\alpha z}^{\prime}}{\partial r_{z}}=\frac{\partial \sigma_{\alpha z}}{\partial r_{z}}+t_{\alpha}$

Integrating over $(z, \infty)$, we obtain

$\sigma_{\alpha z}^{\prime}=\sigma_{\alpha z}-\int_{z}^{\infty} t_{\alpha} d r_{z}$. 


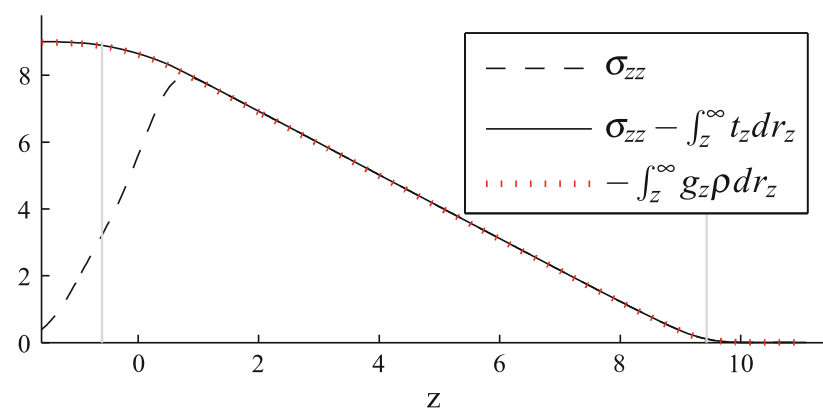

Fig. 3 Downward normal stress $\sigma_{z z}$ without (dashed) and with (solid) correction by the boundary IFD for $w=d / 4$. The stress and IFD exactly match the weight of the flow above height $z$ (red dotted), as expected for steady flows. Gray lines indicate Gray and surface location, calculated using the points where the extended stress definition vanishes and reaches its maximum value (to within $2 \%$ )

Thus, setting $\alpha=z$ in (27), the extended stress component $\sigma_{z z}^{\prime}$ can be obtained without computing the semi-infinite line integral.

The depth profile for the downward normal stress $\sigma_{z z}$ is shown in Fig. 3. Since the rough boundary is not at a fixed height, the stress gradually decreases at the bottom due to the decreasing number of bulk particles near the base. Due to the coarse graining, the stress tensor has a smooth transition even in the case of a flat wall, but the smooth transition disappears as $w \rightarrow 0$. Using the extended stress definition, the bed and surface locations can be defined as the line where the downwards normal stress $\sigma_{z z}^{\prime}$ vanishes and where it reaches its maximum value (to within $2 \%$ ), this is illustrated in Fig. 3; more details can be found Ref. [12]. Additionally, since the flow is steady and uniform, (6) yields the so called lithostatic balance, $\sigma_{z z}^{\prime}=-\int_{z}^{\infty} g_{z} \rho d r_{z}$, which is satisfied with high accuracy.

\section{Conclusions}

We have derived explicit expressions for the stress tensor and the interaction force density (IFD) near an external boundary of a discrete mechanical system. These expressions were obtained by coarse-graining the microscopic equations and therefore exactly satisfy the governing balance laws of mass (5) and momentum (6). A boundary IFD was computed using the contact points between the flow and the basal particles. Our results can be extended to other IFDs, for example, the drag between two different species of particles. The power of our extension to the CG method has been demonstrated by computing stress profiles for a chute flow over a fuzzy boundary. It avoids problems many other methods have near a boundaries. In particular a near-lithostatic stress perpendicular to the wall due to the overlying weight of particle layers is often calculated incorrectly near mildly sloping boundaries.

The present formulation for boundary interaction forces allows us to draw the analogy to electrostatics, where the divergence of the electric field (analogous to the divergence of stress) is compensated by a charge-density source like our interaction force density (21). The analogy can also be made to mixture theory where, by treating the system as a mixture of boundary and flow particles, the IFD is then interpreted as the interaction body force between the two species.

Acknowledgments The authors would like to thank the Institute for Mechanics, Process, and Control, Twente (IMPACT) and the NWOSTW VICI grant 10828 for financial support, and Remco Hartkamp and Dinant Krijgsman for fruitful discussions. The method presented will benefit our research on "Polydispersed Granular Flows through Inclined Channels-Influence of Particle Characteristics, Channel Rotation and Geometry" funded by STW.

Open Access This article is distributed under the terms of the Creative Commons Attribution License which permits any use, distribution, and reproduction in any medium, provided the original author(s) and the source are credited.

\section{References}

1. Goldhirsch, I.: Stress, stress asymmetry and couple stress: from discrete particles to continuous fields. Granul. Mat. 12(3), 239$252(2010)$

2. Frenkel, D., Smit, B.: Understanding Molecular Simulation, 1st edn. Academic Press, San Diego (1996)

3. Cundall, P.A., Strack, O.D.L.: A discrete numerical model for granular assemblies. Geotechnique 29(4765), 47-65 (1979)

4. Luding, S.: Cohesive frictional powders: contact models for tension. Granul. Mat. 10(4), 235-246 (2008)

5. Monaghan, J.J.: Smoothed particle hydrodynamics. Rep. Prog. Phys. 68(8), 1703-1759 (2005)

6. Luding, S., Alonso-Marroquín, F.: The critical-state yield stress (termination locus) of adhesive powders from a single numerical experiment. Granul. Mat. 13(2), 109-119 (2011)

7. Irving, J.H., Kirkwood, J.G.: The statistical mechanical theory of transport processes. J. Chem. Phys. 18, 817-829 (1950)

8. Todd, B.D., Evans, D.J., Daivis, P.J.: Pressure tensor for inhomogeneous fluids. Phys. Rev. E 52(2), 1627-1638 (1995)

9. Babic, M.: Average balance equations for granular materials. Int. J. Eng. Sci. 35(5), 523-548 (1997)

10. Morland, L.W.: Flow of viscous fluid through a porous deformable matrix. Surv. Geophys. 13, 209-268 (1992)

11. Silbert, L.E., Ertas, D., Grest, G.S., Halsey, D., Levine, T.C., Plimpton, S.J.: Granular flow down an inclined plane: Bagnold scaling and rheology. Phys. Rev. E. 64, 051302 (2001)

12. Weinhart, T., Thornton, A.R., Luding, S., Bokhove, O.: Closure relations for shallow granular flows from particle simulations. Granul. Mat. (2011, submitted) 